\title{
Aldosterone induces NRK-52E cell apoptosis in acute kidney injury via rno-miR-203 hypermethylation and Kim-1 upregulation
}

\author{
XIANGCHENG XIAO $^{1}$, RONG TANG $^{1}$, XIAO ZHOU $^{1}$, LING PENG $^{2}$ and PINGPING YU ${ }^{3}$ \\ ${ }^{1}$ Department of Nephrology, Xiangya Hospital; ${ }^{2}$ The Nephrotic Laboratory of Xiangya Hospital; \\ ${ }^{3}$ Department of Geriatrics, Xiangya Hospital, Central South University, Changsha, Hunan 410008, P.R. China
}

Received January 5, 2015; Accepted April 12, 2016

DOI: $10.3892 /$ etm.2016.3443

\begin{abstract}
Acute kidney injury (AKI) is characterized by an acute reduction in kidney function as identified by an increase in serum creatinine levels and reduction in urine output. Kidney injury molecule-1 (Kim-1) is a hallmark of kidney diseases, since it is typically non-detectable in the non-injured kidney, but upregulated and excreted in the urine during AKI. Aldosterone (Aldo) is a mediator of the renin-angiotensin-Aldo system with a pivotal role in the regulation of salt and extracellular fluid metabolism. In the present study, mice subjected to renal ischemia/reperfusion-induced AKI were investigated. The mice exhibited elevated levels of Aldo and angiotensin II, together with increased Kim-1 expression levels in renal tissue. Treatment of the mice with the Aldo receptor antagonist spironolactone decreased Kim-1 expression levels. These results suggest that Aldo may be associated with the expression of Kim-1 during AKI. However, the molecular mechanism underlying the role of Aldo in Kim-1 expression is unclear, and thus was investigated using NRK-52E cells. Aldo was found to induce the apoptosis of NRK-52E cells via the hypermethylation of rno-microRNA (miR)-203 and upregulation of Kim-1. In addition, luciferase reporter assays demonstrated that Kim-1 was a target gene of rno-miR-203 in NRK-52E cells. Furthermore, Aldo-induced NRK-52E cell apoptosis was reduced by treatment with pre-miR-203 and spironolactone to a greater extent when compared with either alone. The results may provide a promising diagnostic marker or novel therapeutic target for AKI.
\end{abstract}

\section{Introduction}

Acute kidney injury (AKI) is a common disorder (complicating $3-50 \%$ of hospital admissions) that is expensive to

Correspondence to: Dr Pingping Yu, Department of Geriatrics, Xiangya Hospital, Central South University, 87 Xiangya Road, Kaifu, Changsha, Hunan 410008, P.R. China

E-mail: pingpingyu2004@163.com

Key words: acute kidney injury, aldosterone, rno-miR-203, hypermethylation, kidney injury molecule-1 manage, prolongs the hospitalization of patients and is associated with increased mortality (1). AKI is characterized by an acute reduction in kidney function, exhibited as an increase in serum creatinine levels and reduction in urine output (2). AKI has been found to be associated with a high morbidity and mortality in up to $22 \%$ of hospitalized patients (3). AKI commonly occurs in patients with acute illness and is a long-recognized complication of cardiac surgery (4). In its most severe form, AKI increases the odds of operative mortality by 3-8 fold $(5,6)$. A number of different events are known to cause AKI, including ischemia, vasoconstrictive drugs, exposure to toxins, hypotension linked to sepsis, and obstruction of the urinary tract, and this condition may lead to various complications, including metabolic acidosis, high potassium levels, uremia, changes in body fluid balance, and damage to and failure of various organs (7). Since the mechanisms underlying AKI are unclear, no specific therapy is currently available to prevent or treat it (8).

Kidney injury molecule-1 (Kim-1) is a transmembrane glycoprotein that typically cannot be detected in the healthy kidney, but which is upregulated and excreted in the urine during the early stages of ischemic or nephrotoxicity-induced proximal tubule injury $(9,10)$. Kim-1 is a marker of the majority of proteinuric, toxic and ischemia-associated kidney diseases. Kim-1 has gained increasing interest due to its potential role in the modulation of tubular damage and repair (11). Previous studies have suggested that Kim-1 may be involved in the differentiation and proliferation of proximal tubule endothelial cells, and may participate in the restoration of renal tubular epithelium tissue, as well as maintaining its functional integrity (11-13). However, at present, the upstream molecular mechanism underlying the dysregulation of Kim-1 expression in AKI is unclear.

Aldosterone (Aldo) is an important mediator of the renin-angiotensin (Ang)-Aldo system (RAAS) and has a pivotal role in the regulation of salt and extracellular fluid metabolism. In addition, Aldo exerts non-hemodynamic effects associated with end organ damage and renal injury (14). These detrimental effects of Aldo are reported to be independent of the RAAS (14). A previous study demonstrated that RAAS induced Kim-1 expression in proximal tubule endothelial cells, leading to kidney injury, whereas treatment with an Aldo receptor antagonist was able to decrease Kim-1 expression and attenuate kidney injury (15). These results suggest that Aldo 
is associated with the expression of Kim-1; however, studies investigating the molecular mechanisms underlying the role of Aldo in Kim-1 expression are lacking.

MicroRNAs (miRNAs) are endogenous RNA molecules, usually 18-25 nucleotides in length, that regulate gene expression at the post-transcriptional level. miRNAs typically target the 3'-untranslated regions (UTRs) of mRNA, and since miRNA target motifs do not require complete homology, there may be multiple mRNA targets for each miRNA (16).

miRNAs are required for normal development, cell growth, differentiation, apoptosis and the regulation of numerous other biological processes in mammals (17). Since they participate in almost every cellular process, their dysregulation has been associated with the development of numerous pathologies, including kidney diseases (18).

miRNAs have been indicated to be key regulators of normal kidney function and development, and are the basis of several renal diseases (18). AKI has been associated with aberrantly expressed miRNAs in several studies; circulating miR-210 predicted improved rates of survival in patients with AKI (19), whereas plasma levels of miR-21 were associated with the progression of AKI (20), and the levels of miR-494 were markedly increased in the urine of patients with AKI (21). Furthermore, some miRNAs have been reported to be novel biomarkers and therapeutic candidates for AKI, including miR-714, miR-1188, miR-1897-3p and miR-182-5p $(22,23)$. However, whether miRNA-mediated dysregulation of Kim-1 occurs has yet to be elucidated.

The present study aimed to investigate the association between the Aldo-induced upregulation of Kim-1 expression and the onset of necrotic and apoptotic cell death in proximal tubule endothelial cells, as well as the potential molecular mechanism underlying the role of miRNAs in AKI.

\section{Materials and methods}

General animal procedures. A total of 24 male CD-1 mice (weight, 25-35 g; aged 6-8 weeks; Xiangya Hospital of Central South University, Changsha, China) were used for the experiments. The mice were maintained under routine vivarium conditions (temperature, $18-26^{\circ} \mathrm{C}$; humidity, $40-70 \%$; light/dark cycle, $10 \mathrm{~h} / 14 \mathrm{~h}$ ) with ad libitum access to food and water throughout. All procedures were performed in accordance with the animal care guidelines at the Xiangya Hospital of Central South University. Ethical approval was provided by the Ethics Committee of the Center for Scientific Research with Animal Models of Central South University. All surgeries were conducted under deep anesthesia, achieved by the intraperitoneal injection of pentobarbital sodium (2-3 mg/mouse; Beijing Solarbio Science \& Technology, Co., Ltd., Beijing, China). During anesthesia and recovery, the body temperature of the mice was maintained at $36-37^{\circ} \mathrm{C}$ using heating lamps during surgery, or by maintaining the mice in a temperature-controlled apparatus during recovery. The mice were divided into four groups, with 6 mice in each group, as follows: Control (con), sham, ischemia/reperfusion (I/R) and I/R + spironolactone (SPIR) groups. The mice in the I/R + SPIR group were administered spironolactone (20 mg/kg/day; Sigma-Aldrich, St. Louis, MO, USA) by gavage 5 days prior to model construction.
Renal I/R model construction. Mice models of renal I/R were established, as described previously (24). Briefly, mice were subjected to a midline abdominal incision, the left renal pedicle was identified and a smooth vascular clamp was applied to it. Following $15 \mathrm{~min}$ of ischemia, reperfusion was confirmed by the attenuation of organ cyanosis. The right kidney was left unperturbed. Following recovery from anesthesia, the mice were returned to their cages. After $48 \mathrm{~h}$ of reperfusion, the mice were reanesthetized, the abdominal incisions were opened and contralateral kidneys were removed. The mice in the sham group were subjected to a midline abdominal incision; however, the kidneys were left unperturbed.

Immunohistochemistry. Renal tissues were obtained, fixed with $4 \%$ formaldehyde solution overnight, dehydrated using an alcohol gradient, cleared using xylene and paraffin-embedded. Subsequently, the paraffin-embedded tissue blocks were sliced into $8 \mathrm{~nm}$ sections using a microtome, which were then mounted onto anti-shedding glass slides. The slides were baked in an oven at $55^{\circ} \mathrm{C}$ for $2 \mathrm{~h}$, after which the sections were deparaffinized and rehydrated, prior to antigen retrieval using sodium citrate. The sections were incubated with rabbit anti-Kim-1 polyclonal antibody (cat. no. ABF199; EMD Millipore, Billerica, MA, USA) or normal rabbit immunoglobulin G (GenWay Biotech, Inc., San Diego, CA, USA) as a negative control overnight at $4^{\circ} \mathrm{C}$. After this, the sections were treated with Universal Immuno-peroxidase Polymer Anti-Mouse/Rabbit Immunohistochemical Staining reagent (PV-8000; ZSGB-Bio, Beijing, China) according to the manufacturer's protocol. Immunostaining was performed using diaminobenzidine (Zhongshan Jinqiao Biological Technology Co., Ltd., Beijing, China) and the stained tissues were examined using the Moticam 3000 system (Motic, Causeway Bay, Hong Kong). Image Pro-Plus software, version 6.0 (Media Cybernetics, Inc., Rockville, MD, USA) was used to quantify the mean density of Kim-1 staining, according to the manufacturer's protocol.

Detection of Aldo by radioimmunoassay (RIA). Urine samples were collected from the mice after $48 \mathrm{~h}$ of reperfusion for the analysis of Aldo by RIA. Prior to analysis, urine samples $(50 \mu \mathrm{l})$ were centrifuged at 3,000 x g for $10 \mathrm{~min}$, after which the supernatant was treated with $\mathrm{HCl}$. Urinary Aldo levels in the supernatant were determined using an Iodine-125 Aldosterone Radioimmunoasssay kit (Shanghai Rongbai Biological Technology, Co., Ltd., Shanghai, China), according to the manufacturer's protocol. The assay tubes were centrifuged at 1,000 x $\mathrm{g}$ for $45 \mathrm{~min}$, after which the supernatant was mixed with $3 \mathrm{ml}$ liquid scintillation fluid (Beckman Coulter, Inc., Brea, CA, USA) prior to counting the radioactivity in an automated $\beta$-counter. Duplicate standard curves with five points ranging from 3 to $800 \mathrm{pg}$ Aldo were obtained. Aldo levels in the urine samples were determined by comparison with the standard curve.

Cell culture and treatment. NRK-52E rat proximal tubular cells (American Type Culture Collection, Manassas, VA, USA) were cultured in Dulbecco's modified Eagle's medium (Sigma-Aldrich) supplemented with $10 \%$ fetal bovine serum, $2 \%$ penicillin-streptomycin (both Gibco; Thermo Fisher 
Scientific, Inc., Waltham, MA, USA), 1\% HEPES buffer, 2 g sodium bicarbonate and $2 \mathrm{mM}$ L-glutamine, in $5 \% \mathrm{CO}_{2}$ at $37^{\circ} \mathrm{C}$, according to a previous study (25). Subsequently, the cells were incubated for $12 \mathrm{~h}$ in an environment containing $94 \% \mathrm{~N}_{2}, 5 \% \mathrm{CO}_{2}$ and $1 \% \mathrm{O}_{2}$ to simulate hypoxia, followed by reoxygenation for $6 \mathrm{~h}$ to generate hypoxia/reoxygenation (H/R) cell models. Other cells were treated with $0.1 \mu \mathrm{mol} / 1$ antimycin A (Santa Cruz Biotechnology, Inc., Dallas, TX, USA) for $24 \mathrm{~h}$ at $30^{\circ} \mathrm{C}$, to create a different fibrotic cell model.

In the subsequent drug treatment experiments, the cells were serum-starved for $24 \mathrm{~h}$, and then treated with either $50 \mathrm{nM}$ Aldo or $100 \mathrm{nM}$ spironolactone (both Sigma-Aldrich) for $1 \mathrm{~h}$ at $30^{\circ} \mathrm{C}$. In addition, when the cells were treated with both spironolactone and Aldo, the cells were pretreated with $100 \mathrm{nM}$ spironolactone for $1 \mathrm{~h}$ prior to the addition of $50 \mathrm{nM}$ Aldo for $1 \mathrm{~h}$.

Cell transfection and dual luciferase reporter assay. In order to analyze the function of miR-203 in AKI, pre-miR-203 or pre-control (FulenGen Co., Ltd., Guangzhou, China) was transfected into NRK-52E cells using Lipofectamine 2000 (Invitrogen; Thermo Fisher Scientific, Inc.), according to the manufacturer's protocol. The 3'-UTR of Kim-1 (NM_173149.2) containing the Rattus norvegicus (rno)-miR-203 binding sites or its corresponding mutated sequence was cloned into the psi-CHECK ${ }^{\mathrm{TM}}$-2 luciferase reporter vector (Promega Corporation, Madison, WI, USA) downstream of the Renilla luciferase, generating Kim-1-3'-UTR and Kim-1-Mut 3'-UTR vectors, respectively. Subsequently, NRK-52E cells were co-transfected with the recombinant reporter constructs and rno-miR-203 mimics, the rno-miR-203 inhibitor, negative control (NC) or NC inhibitor (GeneCopoeia, Guangzhou, China) using Lipofectamine 2000. Luciferase activity was determined after $48 \mathrm{~h}$ using the Dual-Glo ${ }^{\circledR}$ Luciferase Assay system (Promega Corporation) and an LD 400 Luminometer (Beckman Coulter, Inc.). Data are presented as the ratio of experimental (Renilla) luciferase to control (firefly) luciferase.

Reverse transcription-quantitative polymerase chain reaction (RT-qPCR). Total RNA was extracted from cells using TRIzol ${ }^{\circledR}$ reagent (Invitrogen; Thermo Fisher Scientific, Inc.), according to the manufacturer's protocol. The relative expression levels of rno-miR-203 were determined by RT-qPCR using the mirVana ${ }^{\mathrm{TM}}$ qRT-PCR microRNA Detection kit (Ambion; Thermo Fisher Scientific, Inc.) according to the manufacturer's protocol. Specific primer sets for rno-miR-203 (cat. no. RmiRQP0305) and U6 (used as an internal reference; cat. no. HmiRQP9003) were obtained from GeneCopoeia (Rockville, MD, USA). The relative mRNA expression levels of Kim-1 were detected using the standard SYBR Green RT-PCR kit (Takara Bio, Inc., Otsu, Japan), according to the manufacturer's protocol. The specific primer pairs were as follows: Kim-1 sense, 5'-AAGGGCTTCTATGTTGGC-3' and antisense, 5'-CCTCTGGGACTCATTCTG-3'; and $\beta$-actin as an internal control, sense, 5'-CCCATCTATGAGGGT TACGC-3' and antisense, 5'-TTTAATGTCACGCACGAT TTC-3' (Takara Bio, Inc.). The PCR analysis was conducted using an ABI 7300 Real-Time PCR system (Thermo Fisher Scientific, Inc.) with the following cycling conditions: A single cycle at $95^{\circ} \mathrm{C}$ for $3 \mathrm{~min}$, followed by 35 cycles of $95^{\circ} \mathrm{C}$ for $10 \mathrm{sec}$ and $58^{\circ} \mathrm{C}$ for $30 \mathrm{sec}$. The relative expression levels of Kim-1 mRNA or rno-miR-203 were quantified using GraphPad Prism 4.0 software (GraphPad Software, San Diego, CA, USA) and the $2^{-\Delta \Delta \mathrm{Cq}}$ method (26).

Western blotting. Tissues or cells were solubilized in cold radioimmunoprecipitation assay lysis buffer (Auragene Bioscience, Changsha, China) and protein concentrations were determined using a bicinchoninic acid protein assay kit. The proteins $(10 \mu \mathrm{g})$ were separated by $10 \%$ sodium dodecyl sulfate-polyacrylamide gel electrophoresis and then transferred to polyvinylidene difluoride membranes. The membranes were blocked with $10 \%$ non-fat dried milk in phosphate-buffered saline (PBS) containing $0.01 \%$ Tween-20 for $3 \mathrm{~h}$ at room temperature and then incubated overnight at $4^{\circ} \mathrm{C}$ with mouse anti-Kim-1 monoclonal antibody (1:1,000; ab66062; Abcam, Cambridge, MA, USA), mouse anti-Ang II monoclonal antibody (1:1,000; ab9391; Abcam), rabbit anti-caspase-8 polyclonal antibody (1:1,000; AM2288; Abzoom Biolabs, Inc., Dallas, TX, USA), rabbit anti-caspase-9 polyclonal antibody (1:1,000; AM2289; Abzoom Biolabs, Inc.), rabbit anti-cleaved poly (ADP-ribose) polymerase (PARP) polyclonal antibody (1:1,500; sc-23461; Santa Cruz Biotechnology, Inc.) and mouse anti- $\beta$-actin monoclonal antibody (1:2,000; LCA01; Auragene Bioscience). Following subsequent incubation with horseradish peroxidase-conjugated goat anti-rabbit (1:3,000: 111-035-008; Jackson ImmunoResearch Laboratories, Inc., West Grove, PA, USA) and anti-mouse (1:15,000; SA001; Auragene Bioscience) secondary antibodies for $1 \mathrm{~h}$ at room temperature, the immune complexes were detected using an ECL kit (P001WB-1; Auragene Bioscience). Data was analyzed by densitometry using Image-Pro plus software, version 6.0. Each condition was tested in triplicate, and expression levels were normalized to $\beta$-actin expression.

Methylation-specific PCR (MSP). Genomic DNA was extracted from cells or tissues using a Genomic DNA Extraction kit (Takara Biotechnology Co., Ltd., Dalian, China). Genomic DNA (1 $\mu \mathrm{g})$ was modified with bisulfite using the Epitect Bisulfite kit (Qiagen, Inc., Valencia, CA, USA) according to the manufacturer's protocol, and eluted with $40 \mathrm{ml}$ elution buffer. The modified DNA ( $2 \mathrm{ml})$ was then used as the template in a bisulfite PCR reaction. MSP was conducted on the bisulfate-treated DNA using the EZ DNA Methylation-Gold ${ }^{\mathrm{TM}}$ kit (Zymo Research Corporation, Irvine, CA, USA), according to the manufacturer's protocol. Methylation-specific primers for the rno-miR-203 promoter were designed using the MethPrimer program (http://www. urogene.org/methprimer/). Bisulfite-converted genomic DNA was amplified by PCR using methylation-specific primers and the SYBR Green reaction mix (Takara Bio, Inc.). The primers used were as follows: MSP primer forward, 5'-GTCGGTGAT TTAGGGTTATTTTC-3' and reverse, 5'-GACTACACTCCG TACGACGA-3'; and unmethylated-specific primer forward, 5'-GGTTGGTGATTTAGGGTTATTTTT-3', and reverse, 5'-TAACCCAACTACACTCCATACAACA-3'. Cycling was conducted using an ABI 7300 system, with cycling conditions of $94^{\circ} \mathrm{C}$ for $4 \mathrm{~min}$; then 35 cycles of $94^{\circ} \mathrm{C}$ for $30 \mathrm{sec}, 55^{\circ} \mathrm{C}$ for $30 \mathrm{sec}$ and $72^{\circ} \mathrm{C}$ for $30 \mathrm{sec}$; followed by $72^{\circ} \mathrm{C}$ for $5 \mathrm{~min}$. The sample was then maintained at $4^{\circ} \mathrm{C}$. 
A

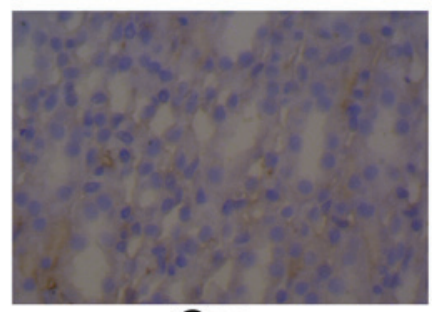

Con

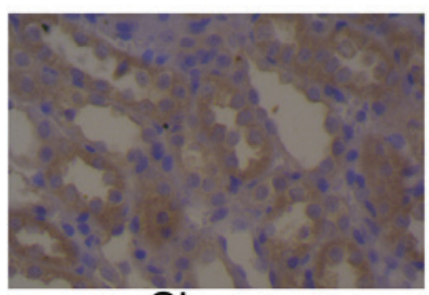

Sham
C

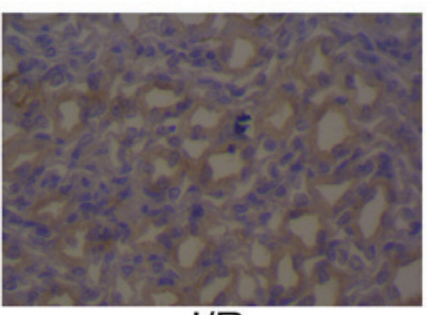

$\mathrm{I} / \mathrm{R}$

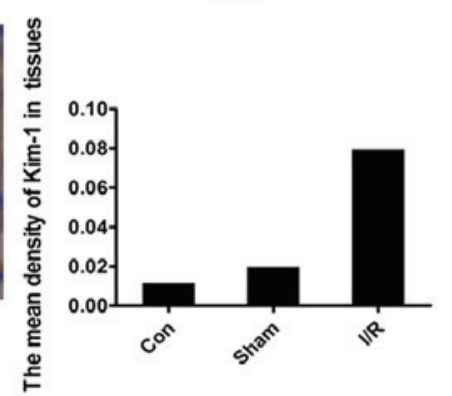

D

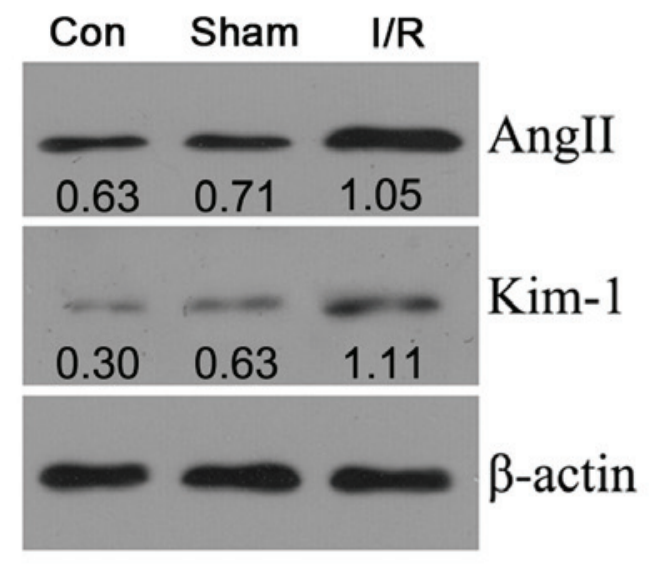

Figure 1. Expression of Kim-1 and Ang II in mice models of I/R-induced acute kidney injury. (A) Representative results of Kim-1 immunohistochemical staining in renal tissues (magnification, x200). (B) Relative mRNA expression levels of Kim-1 in the renal tissues of the various groups were analyzed using reverse transcription-quantitative polymerase chain reaction. (C) Concentration of aldosterone in the urine of the mice was detected using a radioimmunoassay. (D) Protein expression levels of Kim-1 and Ang II in the renal tissues were detected by western blotting. Data are presented as the mean \pm standard deviation ( $\mathrm{n}=3$ ). ${ }^{*} \mathrm{P}<0.05$ vs. the con or sham groups. ${ }^{~} \mathrm{P}<0.05$ between groups. Kim-1, kidney injury molecule-1; Ang II, angiotensin II; I/R, ischemia/reperfusion; SPIR, spironolactone; con, control.

Flow cytometric analysis by annexin V-fluorescein isothiocyanate (FITC) and propidium iodide (PI) double-staining. The apoptosis of NRK-52E cells was analyzed by flow cytometric analysis using the FITC Annexin V Apoptosis Detection Kit I (BD Pharmingen, San Diego, CA, USA) and PI staining, according to the manufacturer's protocols. Briefly, the cells were washed twice with cold PBS and then resuspended in binding buffer. Subsequently, $2 \times 10^{5}$ cells $/ \mathrm{ml}$ were transferred to a $5-\mathrm{ml}$ tube containing $5 \mu 1$ annexin V-FITC and $5 \mu \mathrm{l} \mathrm{PI}$, followed by incubation for $15 \mathrm{~min}$ at room temperature in the dark. Following the addition of $400 \mu \mathrm{l}$ binding buffer, the samples were analyzed by flow cytometry (FACSCalibur; BD Biosciences, Franklin Lakes, NJ, USA) within $1 \mathrm{~h}$. Annexin V-positive and PI-negative cells were defined as apoptotic. Data analysis was performed with ModFit LT software, version 2.0 (Verity Software House, Inc., Topsham, ME, USA).

Bioinformatics. Bioinformatics analysis was conducted using Targetscan (http://www.targetscan.org/) and MicroRNA. org (http://www.microrna.org/microrna/getGeneForm.do) software in order to predict the possibility that a direct target relationship exists between Kim-1 and miR-203. The Li Lab (http://www.urogene.org/) was used to perform the $\mathrm{CpG}$ island prediction.

Statistical analysis. Data are presented as the mean \pm standard deviation of at least three independent experiments. Statistical analyses were performed using SPSS 16.0 software (SPSS, Inc., Chicago, IL, USA). Differences between groups were analyzed by one-way analysis of variance, followed by Fisher's Least Significant Difference test. $\mathrm{P}<0.05$ was considered to indicate a statistically significant difference.

\section{Results}

Expression levels of Kim-1 and levels of Aldo in I/R mice models. In order to investigate Kim-1 expression in AKI renal tissues, I/R mice models were established with or without spironolactone feeding, and urine and renal tissues 
A
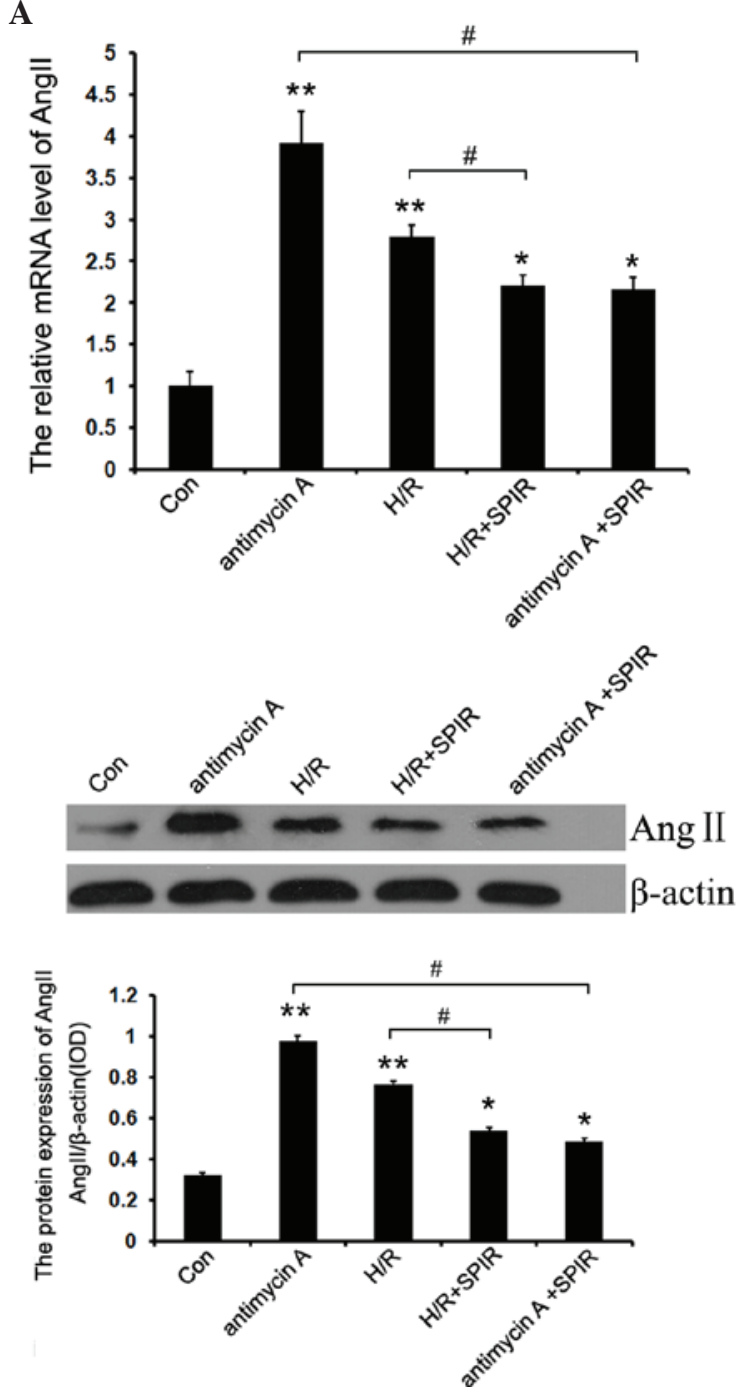

B
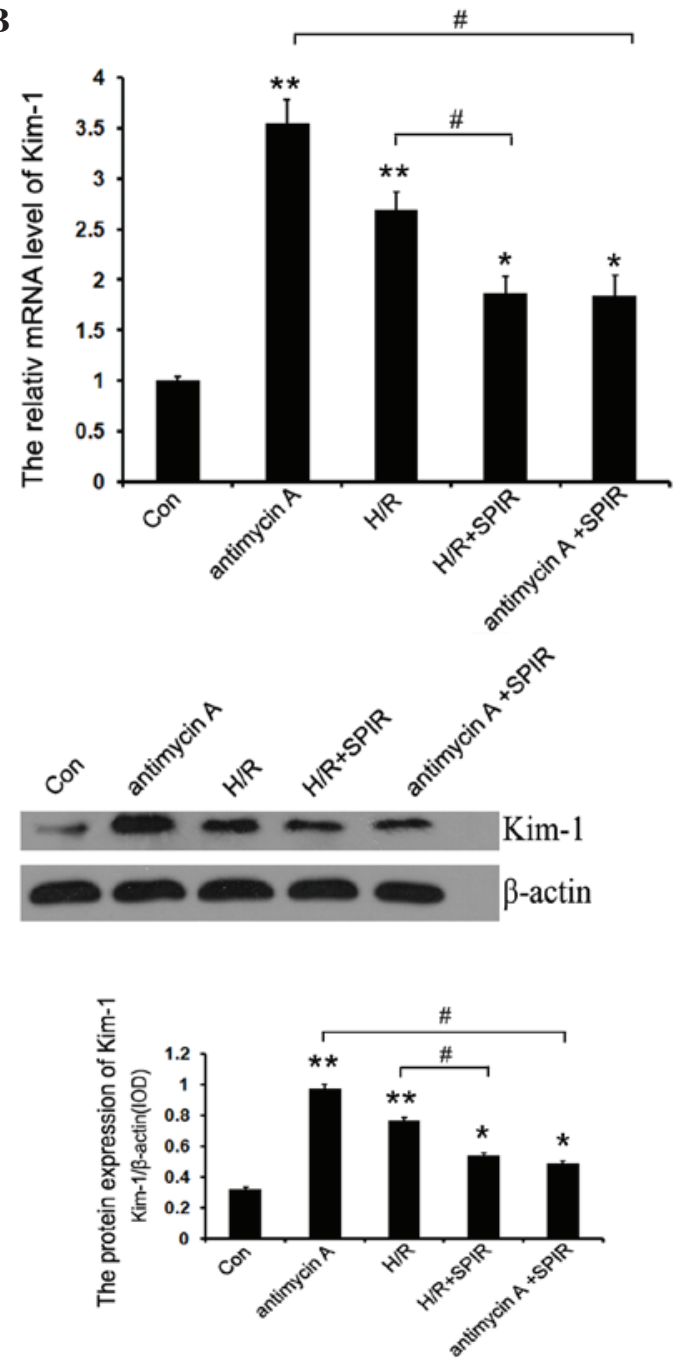

Figure 2. Relative mRNA and protein expression levels of Ang II and Kim-1 in NRK cell models of acute kidney injury. Relative mRNA and protein expression levels of (A) Ang II and (B) Kim-1 in NRK cells treated with H/R or antimycin A with or without SPIR were determined by reverse transcription-quantitative polymerase chain reaction and western blotting. Data are presented as the mean \pm standard deviation $(\mathrm{n}=3) .{ }^{*} \mathrm{P}<0.05,{ }^{* *} \mathrm{P}<0.01$ vs. the con group. ${ }^{\#} \mathrm{P}<0.05$ between groups. Kim-1, kidney injury molecule-1; Ang II, angiotensin II; I/R, ischemia/reperfusion; H/R, hypoxia/reoxygenation; SPIR, spironolactone; con, control; IOD, integrated optical density.

were collected after $48 \mathrm{~h}$. Immunohistochemical analysis was performed in order to detect the expression of Kim-1 in the renal tissues of the various groups and RT-qPCR was conducted to determine the relative mRNA expression levels of Kim-1. Immunohistochemical analyses demonstrated that the expression of Kim-1 was negligible in normal renal tissues; however, it was significantly upregulated in the renal tissues of the group subjected to I/R (Fig. 1A). The mRNA expression level of Kim-1 was markedly lower in the I/R mice treated with spironolactone compared with that in the I/R group (Fig. 1B).

The Aldo levels in the urine of the mice were detected using a RIA. The Aldo level in the urine of the mice in the I/R group was significantly higher compared with that of the control and sham groups $(\mathrm{P}<0.05$; Fig. 1C); however, upon treatment of the $\mathrm{I} / \mathrm{R}$ mice with spironolactone $(20 \mathrm{mg} / \mathrm{kg} / \mathrm{day})$, the Aldo level in the urine of the mice was decreased, although it remained significantly higher than the levels in the control and sham groups $(\mathrm{P}<0.05$; Fig. 1C). These results suggest that elevated Aldo levels may induce Kim-1 expression in the renal tissues of I/R mice. In order to verify these results, western blotting was performed to detect the protein expression levels of Kim-1 and Ang II in the renal tissues from the various groups. The protein expression levels of Kim-1 and Ang II were markedly upregulated in the I/R group, as compared with the control and sham groups (Fig. 1D).

Expression of Ang II and Kim-1 in NRK-52E cell models. In order to establish H/R cell models, NRK-52E cells were subjected to $94 \% \mathrm{~N}_{2}, 5 \% \mathrm{CO}_{2}$ and $1 \% \mathrm{O}_{2}$ to simulate hypoxia, and then reoxygenated. In addition, NRK-52E cells were treated with $0.1 \mu \mathrm{mol} / 1$ antimycin A to obtain a fibrotic cell model. RT-qPCR and western blotting were performed to detect the mRNA and protein expression levels of Kim-1 and Ang II. The mRNA and protein expression levels of Ang II and Kim-1 were significantly upregulated in the $\mathrm{H} / \mathrm{R}$ and antimycin A-treated groups as compared with the control group $(\mathrm{P}<0.01$; Fig. 2). However, in the cells that had been pre-treated with the Aldo inhibitor spironolactone, the mRNA and protein expression levels of Ang 

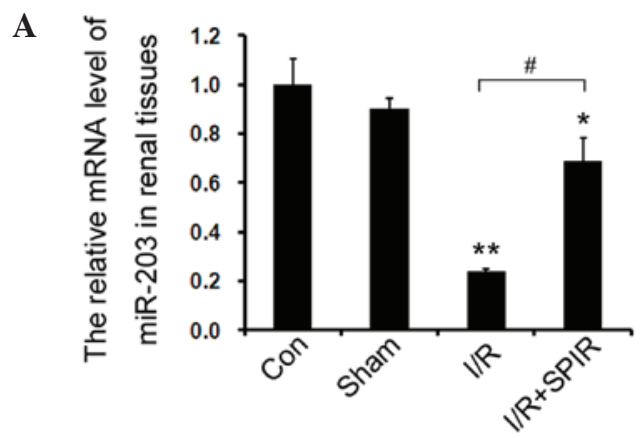

C

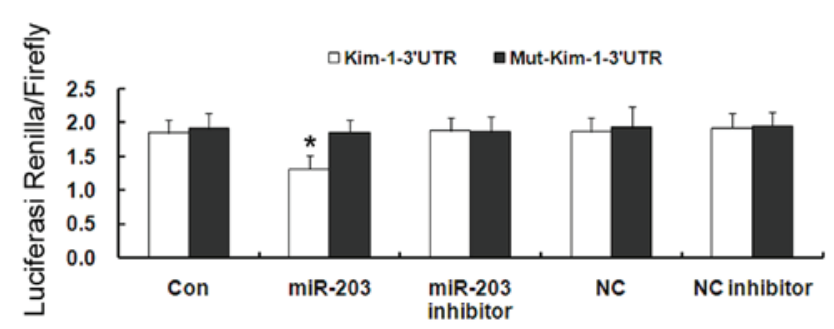

B

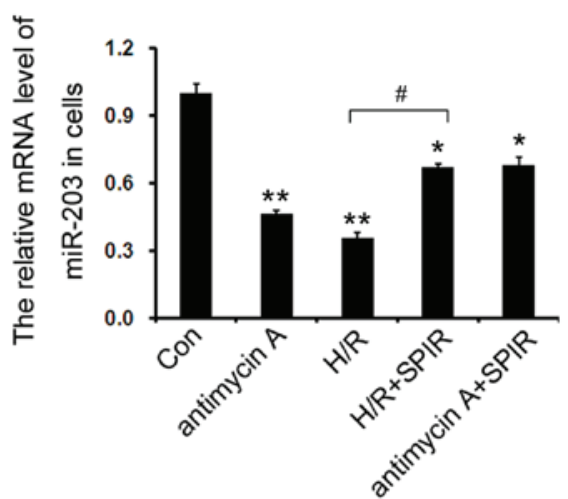

Figure 3. Kim-1 is a direct target of rno-miR-203 in NRK-52E cells. Relative levels of rno-miR-203 in (A) renal tissues of mice in the I/R and I/R + SPIR groups and (B) H/R- and antimycin A-treated NRK cells with or without SPIR determined by reverse transcription-quantitative polymerase chain reaction. ${ }^{*} \mathrm{P}<0.05,{ }^{* *} \mathrm{P}<0.01$ vs. the con group. (C) Dual luciferase reporter assays were conducted to test the interaction of miR-203 and the 3'-UTR of Kim-1, using constructs containing the target sequence (Kim-1-3'UTR) or mutated target sequence (Mut-Kim-1-3'UTR Mut) cloned into psi-CHECK-2 luciferase reporter vectors and transfected into cells. Data are presented as the mean \pm standard deviation ( $\mathrm{n}=3$ ). ${ }^{*} \mathrm{P}<0.05$ vs. the Con group. ${ }^{\#} \mathrm{P}<0.05$ between groups. Kim- 1 , kidney injury molecule-1; miR, microRNA; I/R, ischemia/reperfusion; SPIR, spironolactone; H/R, hypoxia/reoxygenation; con, control; Mut, mutant; 3'-UTR, 3'-untranslated region; NC, negative control.

A

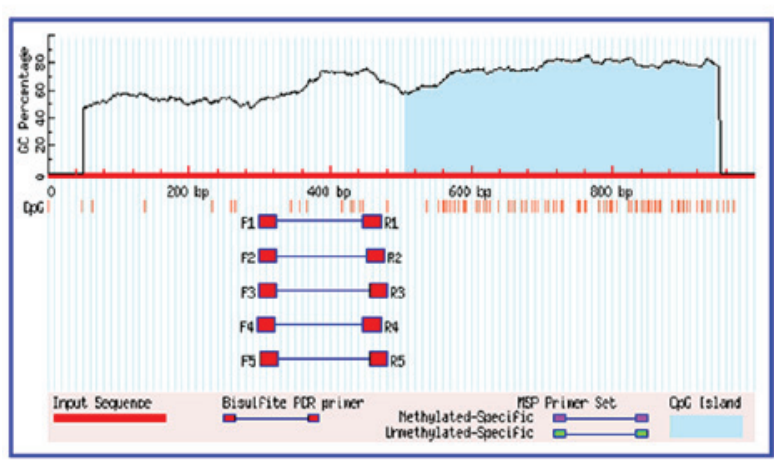

B

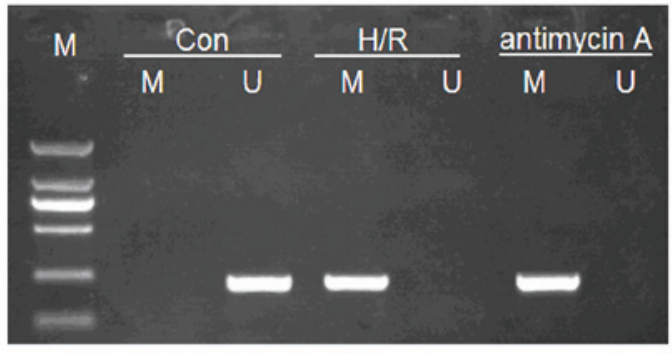

C

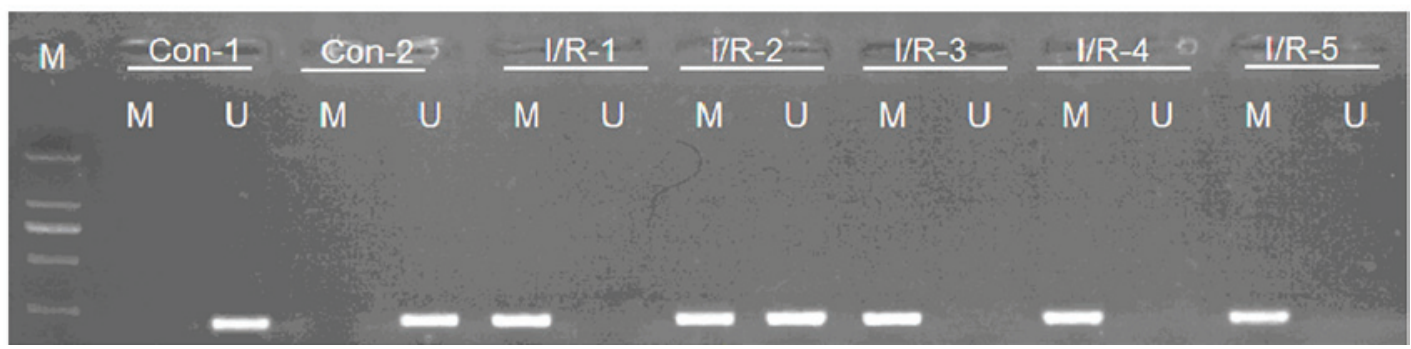

Figure 4. Analysis of the methylation status of miR-203 in the renal tissues of a mouse model of I/R-induced acute kidney injury and in H/R- or antimycin A-treated NRK-52E cell models. (A) The promoter of rno-miR-203 was predicted to encode a CpG island using Li Lab Bioinformatics software; the predicted CpG island is shown in light blue. The methylation status of the rno-miR-203 promoter in (B) NRK cell models and (C) renal tissues of I/R model mice was determined by methylation-specific polymerase chain reaction using specific primers. M, methylated; U, unmethylated; miR, microRNA; I/R, ischemia/reperfusion; H/R, hypoxia/reperfusion.

II and Kim-1 were significantly reduced, as compared with those in the $\mathrm{H} / \mathrm{R}$ and antimycin A-treated groups $(\mathrm{P}<0.05)$, although they remained significantly elevated, as compared with the levels in control cells $(\mathrm{P}<0.05$; Fig. 2). These results suggest that Aldo may have an important role in the upregulation of Kim-1 in $\mathrm{H} / \mathrm{R}$ and antimycin A-treated cell models. 
A
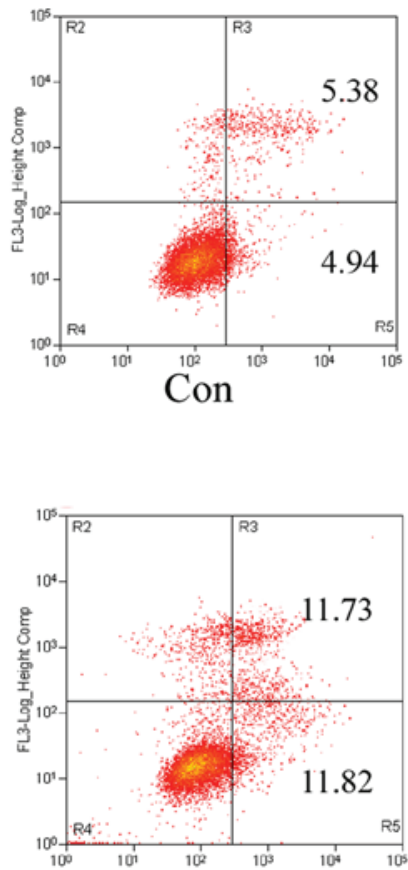

Aldo+pre-miR-203
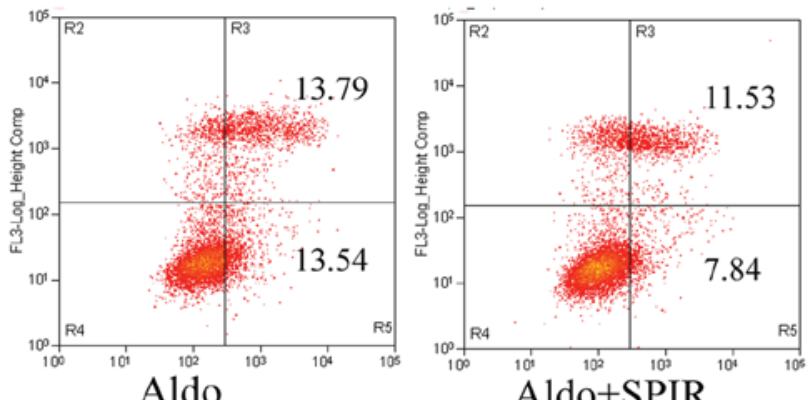

Aldo+SPIR

B
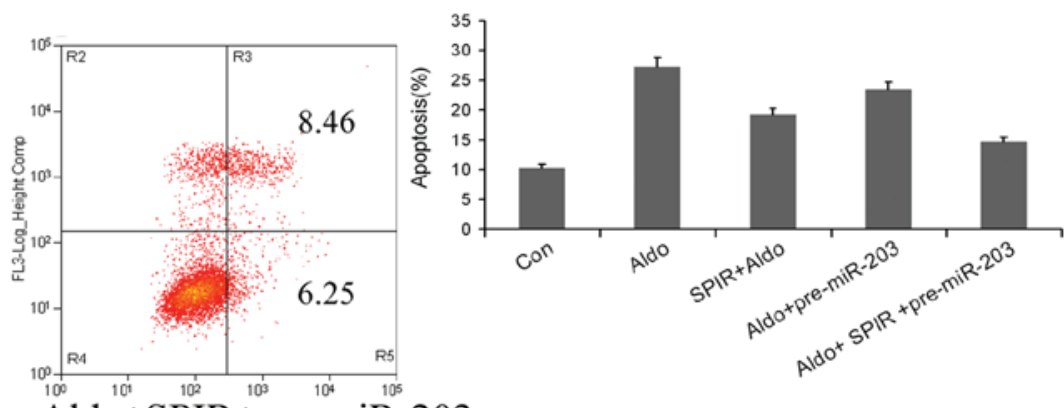

Aldo+SPIR+pre-miR-203

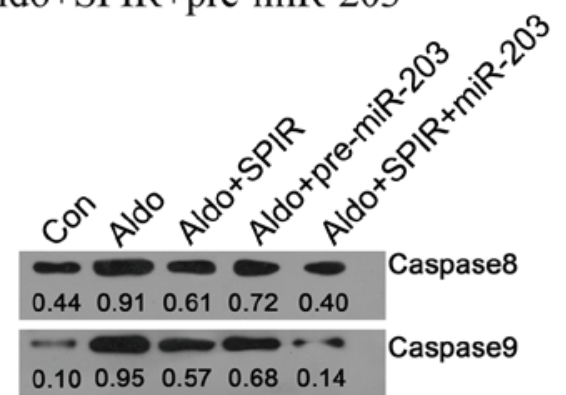

Cleaved PARP

$\begin{array}{lllll}0.27 & 0.82 & 0.47 & 0.61 & 0.18\end{array}$

$\beta$-actin

Figure 5. Effects of rno-miR-203 on NRK cell apoptosis and apoptosis signaling. Cells were treated with Aldo in the presence or absence of SPIR, and then transfected with pre-miR-203. (A) Cell apoptosis was analyzed by flow cytometry. The number in the top right quadrant corresponds to late stage apoptosis, whereas the number in the bottom right quadrant corresponds to early stage apoptosis. Data are presented as the mean \pm standard deviation ( $\mathrm{n}=3$ ). (B) Relative protein expression levels of the apoptosis signaling molecules caspase- 8 , caspase- 9 and cleaved PARP were detected by western blotting; the figures under the bands represent the relative expression levels vs. $\beta$-actin. Aldo, aldosterone; SPIR, spironolactone; miR, microRNA; con, control; PARP, poly(ADP-ribose) polymerase.

Relative expression levels of rno-miR-203 and its association with Kim-1 in NRK cells. Bioinformatics software has predicted that Kim-1 may be a target gene of rno-miR-203. The present study aimed to determine the molecular mechanisms underlying the Aldo-mediated upregulation of Kim-1 expression in NRK-52E cells. qPCR was performed to detect the levels of rno-miR-203 in the renal tissues of the various I/R mouse models and in the cell groups. The levels of rno-miR-203 were downregulated in the renal tissues of the I/R mice, as compared with the control group $(\mathrm{P}<0.01$; Fig. 3A). Conversely, the levels of rno-miR-203 were significantly increased in the renal tissues of the I/R mice treated with spironolactone, as compared with those in the I/R group $(\mathrm{P}<0.05$; Fig. 3A).

The levels of rno-miR-203 were significantly downregulated in the NRK-52E cells treated with H/R or antimycin A as compared with those in normal cells $(\mathrm{P}<0.01$; Fig. $3 \mathrm{~B})$, whereas the levels of rno-miR-203 were markedly increased in the H/R or antimycin A-treated cells pre-treated with spironolactone, as compared with those in the non-pre-treated cells (Fig. 3B). These results suggest that Aldo may have a role in the downregulation of rno-miR-203 in the renal tissues of animals with AKI and in cell models.

In order to assess whether Kim-1 is a direct target of rno-miR-203, luciferase reporter assays were conducted. The Kim-1 3'-UTR fragment containing the rno-miR-203 binding site or a mutated targeting sequence was cloned into a psi-CHECK-2 Dual Luciferase Reporter vector. rno-miR-203 significantly inhibited the luciferase activity in NRK-52E cells transfected with the Kim-1-3'-UTR ( $\mathrm{P}<0.05$; Fig. 3C). However, rno-miR-203 mimics did not suppress the luciferase activity levels in NRK-52E cells transfected with Mut-Kim-1-3'-UTR (Fig. 3C). These results suggest that Kim-1 is a direct target of rno-miR-203 in NRK-52E cells. 
Rno-miR-203 is hypermethylated in AKI tissues and cells treated with $H / R$ or antimycin A. The present study aimed to determine the mechanisms underlying the Aldo-mediated downregulation of rno-miR-203 in AKI tissues and cells. Bioinformatics software predicted that there is a $\mathrm{CpG}$ island in the promoter of rno-miR-203 (Fig. 4A). Therefore, MSP was performed in order to detect the methylation status of the rno-miR-203. MSP demonstrated that both methylated and non-methylated rno-miR-203 were present in the renal tissues of the I/R mice (Fig. 4B), and the NRK-52E cells treated with H/R or antimycin A. Conversely, only non-methylated rno-miR-203 was detected in the control (Fig. 4C). These results suggest that, when AKI occurs, the downregulation of rno-miR-203 may be in part due to its methylation, which in turn may promote the expression of its direct target, Kim-1.

Rno-miR-203 inhibits the apoptosis of NRK-52E cells treated with Aldo. In order to explore the effect of rno-miR-203 on cell apoptosis, pre-miR-203 was transfected into NRK-52E cells and cell apoptosis was analyzed by flow cytometry. Aldo-induced cell apoptosis was reduced in the cells transfected with pre-miR-203, suggesting that miR-203 may have a protective role, similar to that of spironolactone, in preventing cell death induced by high Aldo concentrations. Notably, when the cells were co-treated with pre-miR-203 and spironolactone, the cell apoptosis resulting from exposure to a high Aldo concentration was decreased to a greater extent as compared with that in the groups treated with pre-miR-203 or spironolactone alone (Fig. 5A).

Rno-miR-203 inhibits apoptosis in NRK-52E cells treated with Aldo via death receptor and mitochondrial apoptotic signaling. In order to investigate the mechanism underlying the rno-miR-203-mediated inhibition of Aldo-induced NRK-52E cell apoptosis, western blotting was performed to detect the protein expression levels of the apoptosis initiators, caspase- 8 caspase- 9 and cleaved PARP. The protein expression levels of caspase-8, caspase-9 and cleaved PARP were markedly increased in the cells treated with a high concentration of Aldo. Conversely, when the cells were transfected with pre-miR-203 or pre-treated with spironolactone, the protein expression levels of caspase-8, caspase- 9 and cleaved PARP were markedly decreased compared with those in the Aldo group. In addition, the protein expression levels of caspase- 8 , caspase- 9 and cleaved PARP were most evidently decreased in the cells co-treated with pre-miR-203 and spironolactone (Fig. 5B). These results suggest that rno-miR-203 may attenuate Aldo-induced cell apoptosis via the inhibition of death receptor and mitochondrial apoptotic signaling.

\section{Discussion}

AKI is a common condition in patients who are hospitalized for major surgery or are critically ill, and is associated with increased mortality (27). Previous reports on the molecular mechanisms underlying this disease have been conflicting $(27,28)$. Increasingly, evidence has suggested that apoptosis is the major mechanism underlying early tubule cell death in contemporary clinical AKI $(29,30)$. Nevertheless, controversies remain regarding the contribution of apoptosis to AKI; first, most estimates place the peak incidence of apoptosis at only $3-5 \%$ of tubule cells following ischemic injury, which is arguably insufficient to explain the profound renal dysfunction. Second, apoptosis is more commonly encountered in the distal tubule, whereas loss of viable cells occurs predominantly in proximal segments. Third, apoptosis generally is regarded as a physiological process that removes damaged cells and therefore may be beneficial to the organ and the organism $(29,30)$. Therefore, a comprehensive strategy is required in order to fully delineate the molecular events and pathways underlying the initiation and progression of AKI, as well as to establish a more targeted approach in intervention therapies (3).

Renal ischemia is a common cause of AKI in hospitalized patients. The predominant characteristic of ischemia is hypoxia (26). Antimycin A is a mixture of antimycin compounds that can be used as a respiratory depressant (27). In the present study, mice models of AKI were established by I/R, and NRK-52E cell models of AKI by treatment with $H / R$ or antimycin A.

Aldo is an important mediator of the RAAS and has a pivotal role in the regulation of salt and extracellular fluid metabolism (28). Furthermore, Aldo exerts non-hemodynamic effects, leading to end organ damage and renal injury $(31,32)$. It has previously been reported that the concentration of Aldo in the renal tissues, urine and plasma is increased when AKI occurs, and thus it may be used as a marker of AKI (33). In addition, the Aldo receptor antagonist, spironolactone, has been shown to exert renal protective effects; in a previous study, RAAS-induced Kim-1 expression in proximal tubule endothelial cells led to kidney injury, while treatment with spironolactone reduced Kim-1 expression and attenuated kidney injury (15). These results suggested that Aldo may be associated with the expression of Kim-1 in renal tissues during AKI.

The present study performed immunohistochemical analyses in order to detect the levels of Aldo in the urine of mice. Furthermore, the expression of Ang II, which is the upstream mediator of Aldo in the RAAS (34), was additionally used as a indirect marker of Aldo levels. The concentration of Aldo was upregulated in the urine of the I/R mice models and both Ang II and Kim-1 were upregulated in the I/R mice renal tissues. Conversely, the urine Aldo concentration and Kim-1 expression levels in the renal tissues were significantly reduced in the mice treated with spironolactone compared with those in the $\mathrm{I} / \mathrm{R}$ mice. These results suggest that the upregulation of Kim-1 during AKI was induced by the upregulation of Aldo. Kim-1 was also upregulated in the H/R or antimycin A-treated cell models. However, when the cells were pre-treated with spironolactone, the Kim-1 expression levels were decreased significantly. These results indicated that H/R- or antimycin A-induced renal proximal tubular cell injury was also mediated by Aldo via the upregulation of Kim-1 expression.

The present study aimed to investigate the molecular mechanisms underlying the Aldo-induced upregulation of Kim-1 during AKI, and to determine whether the regulation was the result of direct or indirect processes. Bioinformatics software predicted that Kim-1 may be a target gene of rno-miR-203. Therefore, whether rno-miR-203 is the bridge 
between Aldo and Kim-1 upregulation in AKI was investigated in the present study. The expression levels of rno-miR-203 in the renal tissues of $\mathrm{I} / \mathrm{R}$ mice and $\mathrm{H} / \mathrm{R}$ or antimycin A-treated cells were analyzed by RT-qPCR. The results showed that the levels of rno-miR-203 were significantly reduced in the renal tissues of $\mathrm{I} / \mathrm{R}$ mice compared with those in the controls. In addition, when $\mathrm{I} / \mathrm{R}$ mice were fed with spironolactone, the levels of rno-miR-203 were markedly increased compared with those in the I/R mice. Similar results were observed in the H/R or antimycin A-treated cells. Notably, the pattern of expression of rno-miR-203 was the inverse of that of Kim-1, and a direct association between rno-miR-203 and Kim-1 in NRK-52E cells was verified using luciferase reporter assays.

Bioinformatics software predicted that there is a $\mathrm{CpG}$ island in the promoter of rno-miR-203. Therefore, it was hypothesized that hypermethylation may underlie the dysregulation of rno-miR-203 in AKI, and MSP was performed in order to verify this hypothesis. Hypermethylated rno-miR-203 was detected in the renal tissues of I/R mice and the H/R or antimycin A-treated cells; this suggests that Aldo indirectly induced Kim-1 expression by mediating the hypermethylation of rno-miR-203. In order to reduce Kim-1 expression in NRK cells, the cells were pre-treated with pre-miR-203 and spironolactone prior to treatment with Aldo. Cell apoptosis was significantly reduced in the spironolactone and pre-miR-203-treated groups, as compared with the groups treated with Aldo. Notably, co-treatment with spironolactone and pre-miR-203 had a greater inhibitory effect on apoptosis, as compared with either treatment alone.

In conclusion, the present study demonstrated that Aldo was able to alter the methylation status of rno-miR-203, promoting its hypermethylation and inducing the expression of Kim-1. Kim-1 in turn may induce NRK renal cell apoptosis and necrosis via the death receptor and mitochondrial apoptosis signaling pathways, leading to AKI. To the best of our knowledge, this is the first study to report such findings. The results of the present study suggested that miR-203 may be a promising diagnostic marker or novel therapeutic target for AKI.

\section{Acknowledgements}

The present study was supported by scientific research funds from the Science and Technology Department of Hunan Province (grant no. 2012FJ7001) and the Health Department of Hunan Province (grant no. B2012-012).

\section{References}

1. Dellepiane S, Marengo M and Cantaluppi V: Detrimental cross-talk between sepsis and acute kidney injury: New pathogenic mechanisms, early biomarkers and targeted therapies. Crit Care 20: 61, 2016.

2. Harty J: Prevention and management of acute kidney injury. Ulster Med J 83: 149-157, 2014.

3. Husi H, Sanchez-Niño MD, Delles C, Mullen W, Vlahou A, Ortiz A and Mischak H: A combinatorial approach of proteomics and systems biology in unravelling the mechanisms of acute kidney injury (AKI): Involvement of NMDA receptor GRIN1 in murine AKI. BMC Syst Biol 7: 110, 2013.

4. Xu J, Jiang W, Fang Y, Teng J and Ding X: Management of Cardiac Surgery-Associated Acute Kidney Injury. Contrib Nephrol 187: 131-142, 2016.
5. Ftouh S and Lewington A; Acute Kidney Injury Guideline Development Group convened by the National Clinical Guidelines Centre and commissioned by the National Institute for Health and Care Excellence, in association with The Royal College of Physicians' Clinic: Prevention, detection and management of acute kidney injury: Concise guideline. Clin Med 14: 61-65, 2014.

6. Gaffney AM and Sladen RN: Acute kidney injury in cardiac surgery. Curr Opin Anaesthesiol 28: 50-59, 2015.

7. McKay DB: Intracellular pattern recognition receptors and renal ischemia. Crit Rev Immunol 31: 297-306, 2011.

8. Huang $\mathrm{CH}$, Lai CC, Yang AH and Chiang SC: Myocardial preconditioning reduces kidney injury and apoptosis induced by myocardial ischaemia and reperfusion. Eur J Cardiothorac Surg 48: 382-391, 2015.

9. Prozialeck WC, Edwards JR, Lamar PC, Liu J, Vaidya VS and Bonventre JV: Expression of kidney injury molecule-1 (Kim-1) in relation to necrosis and apoptosis during the early stages of Cd-induced proximal tubule injury. Toxicol Appl Pharmacol 238: 306-314, 2009.

10. Waanders F, Vaidya VS, van Goor H, Leuvenink H, Damman K, Hamming I, Bonventre JV, Vogt L and Navis G: Effect of renin-angiotensin-aldosterone system inhibition, dietary sodium restriction, and/or diuretics on urinary kidney injury molecule 1 excretion in nondiabetic proteinuric kidney disease: A post hoc analysis of a randomized controlled trial. Am J Kidney Dis 53: 16-25, 2009.

11. Waanders F, van Timmeren MM, Stegeman CA, Bakker SJ and van Goor H: Kidney injury molecule-1 in renal disease. J Pathol 220: 7-16, 2010.

12. Bonventre JV: Kidney injury molecule-1 (KIM-1): A urinary biomarker and much more. Nephrol Dial Transplant 24: 3265-3268, 2009.

13. Han WK, Waikar SS, Johnson A, Betensky RA, Dent CL, Devarajan P and Bonventre JV: Urinary biomarkers in the early diagnosis of acute kidney injury. Kidney Int 73: 863-869, 2008.

14. Chen C, Liang W, Jia J, van Goor H, Singhal PC and Ding G: Aldosterone induces apoptosis in rat podocytes: Role of PI3-K/Akt and p38MAPK signaling pathways. Nephron Exp Nephrol 113: e26-e34, 2009.

15. de Borst MH, van Timmeren MM, Vaidya VS, de Boer RA, van Dalen MB, Kramer AB, Schuurs TA, Bonventre JV, Navis G and van Goor H: Induction of kidney injury molecule-1 in homozygous Ren 2 rats is attenuated by blockade of the renin-angiotensin system or p38 MAP kinase. Am J Physiol Renal Physiol 292: F313-F320, 2007.

16. Vrba L, Jensen TJ, Garbe JC,Heimark RL, Cress AE, Dickinson S, Stampfer MR and Futscher BW: Role for DNA methylation in the regulation of miR-200c and miR-141 expression in normal and cancer cells. PloS One 5: e8697, 2010.

17. Kusenda B, Mraz M, Mayer J and Pospisilova S: Re. Biomed Pap Med Fac Univ Palacky Olomouc Czech Repub 150: 205-215, 2006.

18. Aguado-Fraile E, Ramos E, Conde E, Rodríguez M, Liaño F and García-Bermejo ML: MicroRNAs in the kidney: Novel biomarkers of acute kidney injury. Nefrologia 33: 826-834, 2013.

19. Lorenzen JM, Kielstein JT, Hafer C, Gupta SK, Kümpers P, Faulhaber-Walter R, Haller H, Fliser D and Thum T: Circulating miR-210 predicts survival in critically ill patients with acute kidney injury. Clin J Am Soc Nephrol 6: 1540-1546, 2011.

20. Du J, Cao X, Zou L, Chen Y, Guo J, Chen Z, Hu S and Zheng Z: MicroRNA-21 and risk of severe acute kidney injury and poor outcomes after adult cardiac surgery. PloS One 8: e63390, 2013.

21. Lan YF, Chen HH, Lai PF, Cheng CF, Huang YT, Lee YC, Chen TW and Lin H: MicroRNA-494 reduces ATF3 expression and promotes AKI. J Am Soc Nephrol 23: 2012-2023, 2012.

22. Bellinger MA, Bean JS, Rader MA, Heinz-Taheny KM, Nunes JS, Haas JV, Michael LF and Rekhter MD: Concordant changes of plasma and kidney microRNA in the early stages of acute kidney injury: Time course in a mouse model of bilateral renal ischemia-reperfusion. PloS One 9: e93297, 2014.

23. Wilflingseder J, Sunzenauer J, Toronyi E, Heinzel A, Kainz A, Mayer B, Perco P, Telkes G, Langer RM and Oberbauer R: Molecular pathogenesis of post-transplant acute kidney injury: Assessment of whole-genome mRNA and miRNA profiles. PloS One 9: e104164, 2014

24. Zager RA, Johnson AC, Hanson SY and Lund S: Ischemic proximal tubular injury primes mice to endotoxin-induced TNF-alpha generation and systemic release. Am J Physiol Renal Physiol 289: F289-F297, 2005. 
25. Tokumoto M, Lee JY, Fujiwara Y and Satoh M: Alteration of DNA binding activity of transcription factors in NRK-52E rat proximal tubular cells treated with cadmium. J Toxicol Sci 39: 735-738, 2014.

26. Arocho A, Chen B, Ladanyi M and Pan Q: Validation of the 2-DeltaDeltaCt calculation as an alternate method of data analysis for quantitative PCR of BCR-ABL P210 transcripts. Diagn Mol Pathol 15: 56-61, 2006.

27. Husi $\mathrm{H}$ and Human C: Molecular determinants of acute kidney injury. J Inj Violence Res 7: 75-86, 2015.

28. Devarajan P: Update on mechanisms of ischemic acute kidney injury. J Am Soc Nephrol 17: 1503-1520, 2006.

29. Dagher PC: Apoptosis in ischemic renal injury: Roles of GTP depletion and p53. Kidney Int 66: 506-509, 2004.

30. Kaushal GP, Basnakian AG and Shah SV: Apoptotic pathways in ischemic acute renal failure. Kidney Int 66: 500-506, 2004.
31. López-Andrés N, Iñigo C, Gallego I, Diez J and Fortuño MA: Aldosterone induces cardiotrophin-1 expression in HL-1 adult cardiomyocytes. Endocrinology 149: 4970-4978, 2008.

32. White LE, Hassoun HT, Bihorac A, Moore LJ, Sailors RM, McKinley BA, Valdivia A and Moore FA: Acute kidney injury is surprisingly common and a powerful predictor of mortality in surgical sepsis. J Trauma Acute Care Surg 75: 432-438, 2013.

33. Sawada H, Naito Y, Oboshi M, Iwasaku T, Okuhara Y, Morisawa D, Eguchi A, Hirotani S and Masuyama T: Iron restriction inhibits renal injury in aldosterone/salt-induced hypertensive mice. Hypertens Res 38: 317-322, 2015.

34. Chun TY, Chander PN, Kim JW, Pratt JH and Stier CT Jr: Aldosterone, but not angiotensin II, increases profibrotic factors in kidney of adrenalectomized stroke-prone spontaneously hypertensive rats. Am J Physiol Endocrinol Metab 295: E305-E312, 2008. 